\title{
CD3-negative Lymphoproliferative Disease of Granular Lymphocytes Containing Epstein-Barr Viral DNA
}

\author{
Keisei Kawa-Ha, Shigehiko Ishihara, Tsuneo Ninomiya, " Keiko Yumura-Yagi, Junichi Hara, \\ Fumihide Murayama, Akio Tawa, and Kanji Hirai ${ }^{\ddagger}$ \\ Department of Pediatrics, Osaka University Hospital, 1-1-50, Fukushimaku, Osaka 553, Japan; *Department of Pediatrics, \\ Tokushima University Hospital, Tokushima 770, Japan; and $\ddagger^{\ddagger}$ Department of Molecular Biology, \\ Tokai University Medical School, Isehara 259-11, Japan
}

\begin{abstract}
Lymphoproliferative disease of granular lymphocytes (LDGL) is a heterogeneous disorder and the pathogenesis is likely to be complex. Some patients with chronic active EBV (CAEBV) infection also have LDGL. To investigate the relationship between EBV infection and the pathogenesis of LDGL, we conducted a survey for EBV DNA sequences by Southern blot analysis of DNA obtained from the peripheral blood of seven patients with LDGL, including one with CAEBV infection. Interestingly, EBV DNA was detected in the sample from the patient with CAEBV infection, and in the samples from four other patients with CD3- LDGL. Moreover, a single band for the joined termini of the EBV genome was demonstrated in two samples, suggesting a clonal disorder of those LDGL. These findings strongly suggest that EBV may play a pathogenic role in some cases of LDGL.
\end{abstract}

\section{Introduction}

Large granular lymphocytes, which are characterized by the presence of azurophilic granules in their cytoplasm, are the principal effectors of natural killer function and antibody-dependent, cell-mediated cytotoxicity, and are involved in the protection of the host against infections and tumor growth (1-3). Recently, an increasing number of patients showing lymphocytosis of peripheral blood and bone marrow granular lymphocytes have been reported. Given the heterogeneity of the clinical, phenotypic, and functional findings as well as genotypes, the pathogenesis of lymphoproliferative disease of granular lymphocytes (LDGL) ${ }^{1}$ is likely to be complex, ranging from reactive conditions to aggressive malignancies $(4,5)$.

On the other hand, EBV has been implicated as the causative agent in classical infectious mononucleosis (IM) and has

Address correspondence to Dr. Keisei Kawa-Ha, Department of Pediatrics, Osaka University Hospital, 1-1-50, Fukushimaku, Osaka 553, Japan.

Received for publication 11 November 1988 and in revised form 6 March 1989.

1. Abbreviations used in this paper: CAEBV, chronic active EBV; EA, early antigens; IM, infectious mononucleosis; LDGL, lymphoproliferative disease of granular lymphocytes; TCR, T cell receptor; VCA, viral capsid antigen.

J. Clin. Invest.

(C) The American Society for Clinical Investigation, Inc. $0021-9738 / 89 / 07 / 0051 / 05 \$ 2.00$

Volume 84, July 1989, 51-55 been associated with Burkitt's lymphoma and nasopharyngeal carcinoma (6). More recently, it has been recognized that a minority of patients develop chronic active EBV (CAEBV) infection, which is typified by protracted or recurrent IM-like symptoms and an unusual pattern of anti-EBV antibodies (increased anti-early antigens [EA] and/or absent anti-nuclear antigen) $(7,8)$. Furthermore, although it is apparently unusual, some patients with CAEBV develop LDGL (9).

Based on these findings, and because the currently available data don't demonstrate that EBV is causally involved in LDGL, we conducted a survey for EBV DNA sequences by Southern blot analysis of DNA obtained from the peripheral blood of patients with LDGL.

Here we report evidence that EBV DNA was present in five of seven samples, and that two samples containing EBV DNA were of clonal origin.

\section{Methods}

Seven patients (four males and three females) were studied. The clinical and serological findings of the patients are summarized in Table I. All patients had an increased percentage of granular lymphocytes. Splenomegaly and neutropenia were detected in five patients (patients 1-5), and anemia in five (patients 1, 3, 4-6). These characteristics were compatible with $\operatorname{LDGL}(4,5,10)$. Clinically, these seven patients were divided as follows: two chronic, two aggressive, and three progressive after a couple of years of a chronic course. Patients 6 and 7 have been reported elsewhere $(11,12)$. Samples were obtained from peripheral blood, and the cell morphology was evaluated by May-GrunwaldGiemsa staining.

$E B V$ serology. Antibodies to the EB viral capsid antigen, EA, and nuclear antigen were detected by the indirect immunofluorescence method as previously described $(13,14)$.

Immunologic phenotype. Mononuclear cells were obtained after Ficoll-Hypaque gradient centrifugation. Phenotypic analysis using a panel of MAbs such as CD2/T11, CD3/T3, CD4/T4, CD8/T8, CD20/ B1, HLA-DR/I2, CD16/Leu-11, NKH-I, and CD7/Leu-9 was carried out as described previously (15).

DNA studies. Genomic DNA was extracted from the mononuclear cells and was digested with Eco RI and Bam HI restriction endonucleases. The digested DNA was electrophoresed through $0.8 \%$ agarose gels and transferred to nylon membranes. Blots were hybridized to probes labeled with ${ }^{32} \mathrm{P}$ by the random primer method (16). The presence of EBV-specific DNA sequences was determined by using Bam HI-W (Bam W) fragments of the EBV (B95-8 isolate) DNA from a pBR322EBV recombinant library $(17,18)$, and the polymorphic fused termini of the EBV genome were analyzed by using a probe consisting of a 4.6-kb Bam HI-Eco RI fragment containing the tandem terminal repeated sequences of the EBV genome (19), which provides a virus-specific, clonotypic marker for the clonal pathogenesis of EBV-associated tumors. The probe was derived from circularized plasmid EBV DNA (20) and donated by Dr. E. Kieff (Department of Medicine, Harvard University). For analysis of $T$ cell receptor (TCR) gene rearrangements, TCR $\beta$-gene probe (the Bgl II-Eco RV fragment of the cDNA 
Table I. Clinical and Serological Findings of Seven Patients

\begin{tabular}{|c|c|c|c|c|c|c|c|c|c|}
\hline \multirow[b]{2}{*}{ Patient } & \multirow[b]{2}{*}{ Age/sex } & \multirow{2}{*}{$\begin{array}{l}\text { White blood } \\
\text { cells }\end{array}$} & \multirow[b]{2}{*}{ Lymphocytes } & \multirow[b]{2}{*}{$\mathrm{GL}^{*}$} & \multirow[b]{2}{*}{ Splenomegaly } & \multicolumn{3}{|c|}{ EBV antibody titers } & \multirow[b]{2}{*}{ Clinical course } \\
\hline & & & & & & VCA IgG & $\operatorname{EA}(D)^{\ddagger} \operatorname{Ig} G$ & EBNA $^{8}$ & \\
\hline & & $\times 10^{9} /$ liter & $\%$ & $\%$ & & & & & \\
\hline 1 & $13 / \mathrm{M}$ & 3.7 & 63 & 52 & + & 640 & 160 & 320 & Chronic to progressive for $3 \mathrm{yr}$ \\
\hline 2 & $30 / \mathrm{F}$ & 13.3 & 99 & 84 & + & 20 & $<10$ & $\mathrm{NA}^{\| \prime}$ & Aggressive \\
\hline 3 & $7 / F$ & 10.2 & 60 & 40 & + & 20 & $<10$ & 40 & Chronic for $2 \mathrm{yr}$ \\
\hline 4 & $15 / \mathrm{M}$ & 7.1 & 90 & 76 & + & 160 & $<10$ & 80 & Aggressive \\
\hline 5 & $16 / \mathrm{F}$ & 4.8 & 78 & 66 & + & 640 & $<10$ & 40 & Chronic to progressive for $3 \mathrm{yr}$ \\
\hline 6 & $65 / M$ & 11.4 & 90 & 60 & - & 80 & NA & 80 & Chronic for $4 \mathrm{yr}$ \\
\hline 7 & $69 / \mathrm{M}$ & 16.4 & 90 & 85 & - & 160 & $<10$ & 20 & Chronic for $4 \mathrm{yr}$ \\
\hline
\end{tabular}

* Granular lymphocytes; ${ }^{\ddagger}$ early antigen (diffuse); ${ }^{\S}$ EB nuclear antigen; " not assayed.

clone YT-35, containing the constant region of the TCR $\beta$-gene [21]) and the human TCR $\gamma$-gene probe (the Eco RI-Hind III fragment of the cDNA clone M13H60, containing the joining region of the TCR $\gamma$-gene [22]) were used, as described previously (15).

\section{Results}

As shown in Table I, all seven patients had antibodies to viral capsid antigen (VCA) and nuclear antigen, indicative of past infection $(13,14)$. Patient 1 , who was diagnosed as having CAEBV infection two years ago, had an elevated level of IgG antibodies to EA as well as IgG antibodies to VCA and antibodies to nuclear antigen at varying times. Other antibodies such as anti-VCA IgM and IgA, or anti-EA IgM and IgA were not detected. Antibodies to human T cell leukemia virus type I were negative for all samples (data not shown) (23).

All samples expressed $\mathrm{CD} 2$ antigen, while the expression of CD16 and HLA-DR antigens was variable, which is consistent with recent findings (Table II). In contrast, only patient 7 revealed CD3+LDGL, which is the more common type of LDGL according to the previous reports $(4,5)$.

Patients 1-6, who had CD3-LDGL, demonstrated the germline configurations for the TCR $\beta$ - and $\gamma$-genes, while patient 7, who had CD3+LDGL, showed rearrangements of the TCR $\beta$ - and $\gamma$-genes, indicating clonal T cell proliferation (data not shown). To detect EBV DNA, EBV Bam W probe was used. As shown in Fig. 1, a major band at $3 \mathrm{~kb}$, which corresponds to the Bam W fragments of EBV, was demonstrated for patients 1-5 as well as for DNA from an EBV-positive B cell line, Jijoye (lane $C$ ). No bands were observed in lanes $T, 6$, or 7 . Lane $T$ in panel $A$ contained thymocyte DNA used as the negative control and did not reveal any bands. In parallel experiments we studied 15 patients with acute IM and were unable to detect EBV DNA in their peripheral blood, as reported by Andiman et al. (17).

Fig. 2 shows the results of hybridization with a probe specific for one of the termini of the EBV genome. The samples from patients 3 and 4 containing EBV DNA demonstrated a single band, indicating a monoclonal disorder of their LDGL. A convincing result was not available for patient 5 in terms of clonality.

\section{Discussion}

Our results showed that EBV DNA was present in the PBMC obtained from five of the seven patients with LDGL. Since most of lymphocytes were granular lymphocytes in patients 2 ,

Table II. Phenotypes, Genotypes, and EBV DNA in Seven Patients with LDGL

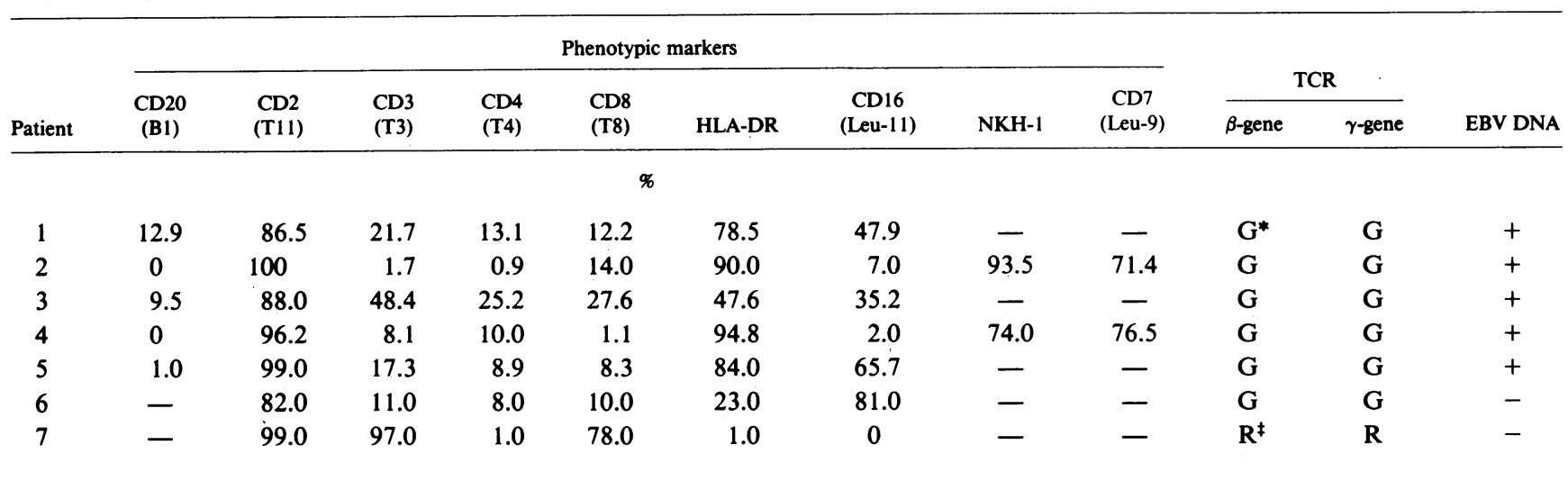

* Germline configuration; ${ }^{\ddagger}$ rearranged. 

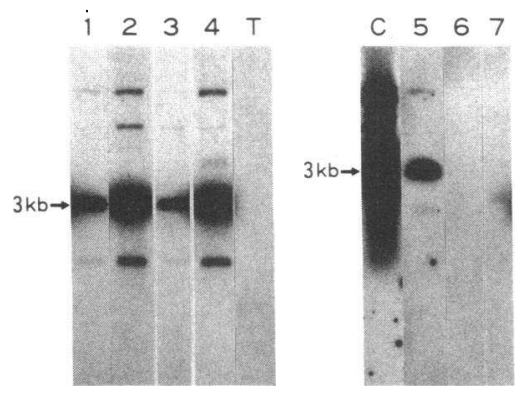

Figure 1. Southern blot ( $5 \mu \mathrm{g}$ of each DNA digested with Bam $\mathrm{HI}$ ) showing the presence of a high number of EBV genomes in lanes $1-5$ (patients 1-5), as well as lane $C$ (EBV-positive B cell line, Jijoye), which was used as a positive control. No hybridization bands were ob-

served in lanes 6,7 , or $T$. Lane $T$ contained thymocyte DNA as a negative control. The Bam W probe detected a 3-kb Bam HI fragment derived from EBV DNA (arrow).

4 , and 5, and no CD20+ cells were detected in patients 2 and 4 (Table II), we surmised that the detected EBV DNA in these patients with LDGL arose from granular lymphocytes. Although we, as well as Andiman et al., were unable to detect EBV DNA in the peripheral blood from patients with acute IM by the method used in the present study, EBV DNA has been found in B lymphocytes. Therefore, it is still possible to argue that a few contaminating $B$ lymphocytes might have affected our results. To check this, we sorted $\mathrm{CD} 20+$ cells from the peripheral blood of patient 1 using a cytometer (Flow Laboratories, Inc., McLean, VA) with a Consort 30-based computer system (FACS; Becton Dickinson Immunocytometry Systems, Mountain View, CA). The DNA sample from CD20- cells again revealed the same band at $3 \mathrm{~kb}$ (data not shown). To further elucidate this we carried out two-color analysis according to our previous report (14). Because granular lymphocytes from patient 1 also expressed NKH-1, we analyzed the patient's cells using a combination of NKH-1-PE vs. Leu-11-FITC. As shown in Fig. 3, a majority of granular lymphocytes from patient 1 , who previously received splenectomy due to progressive thrombocytopenia and neutropenia, expressed both Leu-11 and NKH-1. Since EBV can infect lymphoid cells through the C3d (CR2) receptor that is recognized by $B 2$ antibody $(24,25)$, we used a combination of B2-PE vs.

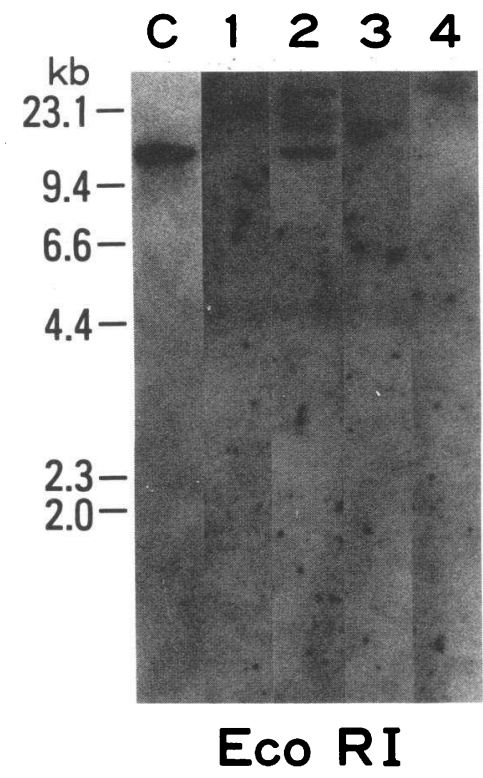

Figure 2. The samples from patients 3 and 4 (lanes 3 and 4 ) demonstrated a single band after digestion with Eco RI and hybridization with the probe recognizing the termini of the EBV genome, indicating a clonal disorder. The other lanes ( 1 and 2) show more than one band, indicating oligo- to polyclonal proliferations of the given cells. Lane $C$ contained a clonal EBV-positive $B$ cell line.
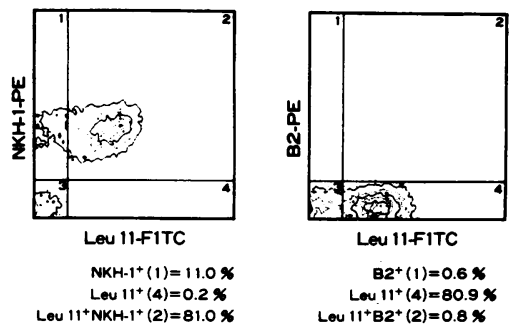

Figure 3. A majority of granular lymphocytes from patient 1 had dual markers of Leu-11+NKH-1+ $(81 \%)$. However, B2 antigen (C3d receptor) was not expressed in these Leu- $11+$ cells.

Leu-11-FITC to investigate the expression of B2 in these Leu-11+ cells. As shown in Fig. 3, we failed to demonstrate the expression of B2 on the cell surface of Leu-11+ cells, suggesting modulation of the receptor for EBV in those EBV-infected cells or the existence of other yet-unknown mechanisms of EBV transmission. After sorting the Leu-11+ cells, DNA was extracted and Southern blot analysis was performed. As shown in Fig. 4, EBV DNA was similarly demonstrated in the DNA sample from the Leu-11+ cells, supporting our present results. However, the Leu-11 - cell sample also showed a 3-kb band (Fig. 4). Based on the phenotypic analyses, the Leu-11- cells mainly consisted of CD3 + T cells (7\%) with $<1 \%$ of CD20+ B cells and monocytes. Similar to previous reports, and our own observation in which EBV DNA was demonstrated in T cells (26-28), those $T$ cells in patient 1 might have been infected by EBV. If so, the infected $T$ cells should be polyclonal, because the EBV termini probe recognized at least two bands (Fig. 2). Moreover, the Leu-11- cell sample revealed only germline configuration of TCR $\beta$ - and $\gamma$-genes (Fig. 5), supporting the above speculation.

The clonal nature of LDGL has been established in some but not all cases of LDGL in terms of TCR gene rearrangement $(4,5)$. All six patients with CD3-LDGL in the present study showed the germline configurations of TCR $\beta$ - and $\gamma$ genes (data not shown, Table II), thus providing no evidence of the clonal origin of the expanded cells. However, the analysis of EBV-genome termini clearly demonstrated a clonal proliferation in patients 3 and 4 indicating a neoplastic, rather than reactive, condition of their $\mathrm{LDGL}$.

In the present study, using the hybridization technique we found for the first time that granular lymphocytes from patients with LDGL contained detectable EBV DNA. Moreover,

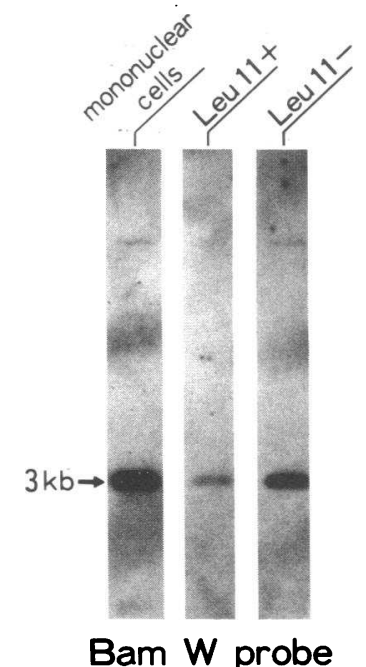

Figure 4. After sorting the Leu-11+ cells from the patient 1 sample, DNA was extracted from each subpopulation. Similar to the unsorted sample, the sorted samples from both the Leu-11+ cells and the Leu-11 - cells demonstrated a 3-kb band. A weak intensity band seen in lane labeled Leu-11+ is due to limited availability of DNA from the sorted cells. 


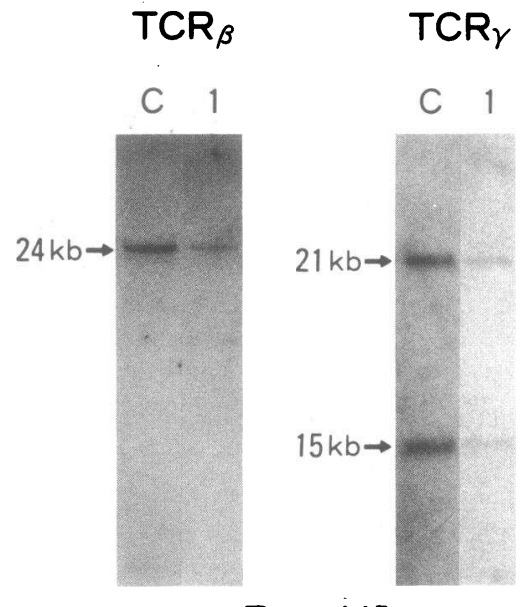

Bam HI
Figure 5. The Leu-11cell sample from patient 1 showing germline configurations of TCR $\beta$ - and $\gamma$-genes.

we were able to show the clonal nature of those increased granular lymphocytes despite the absence of clonal TCR gene rearrangements.

These findings raise the possibility of an etiologic link between EBV infection and some instances of LDGL with a CD3 - phenotype, and also provide a further step in our understanding of the heterogeneity of LDGL.

In the literature $(4,5,10)$, the clinical course of most patients had been stable without any treatment, and the most frequently observed phenotype was CD3+LDGL. In the present study, however, six of the seven patients were of CD3-LDGL, and three patients (patients 2, 4, and 5) died of LDGL. It remains unknown whether the clinical course of the CD3-LDGL might be more aggressive, or the presence and monoclonality of EBV DNA might correlate with the outcomes. To clarify this issue, survey for EBV DNA in more cases of LDGL seems to be indicated.

\section{Acknowledgments}

The authors would like to thank Drs. S. Tagawa, C. Shimazaki, and T. Kato for providing samples for this study. We also thank Dr. T. W. Mak for the YT-35 probe and Dr. T. H. Rabbitts for the M13H60 probe.

This work was supported by the Ministry of Education, Science and Culture of Japan, the Ministry of Health and Welfare of Japan, and the Children's Cancer Association of Japan.

\section{References}

1. Timonen, T., J. R. Ortaldo, and R. B. Herberman. 1981. Characteristics of human large granular lymphocytes and relationship to natural killer and K cells. J. Exp. Med. 153:569-582.

2. Trinchieri, G., and B. Perussia. 1984. Human natural killer cells: biologic and pathologic aspects. Lab. Invest. 50:489-513.

3. Pandolfi, F., G. De Rossi, G. Semenzato, I. Quinti, A. Ranucci, G. De Sanctis, M. Lopez, G. Gasparotto, and F. Aiuti. 1982. Immunologic evaluation of $\mathrm{T}$ chronic lymphocyte leukemia cells. Correlations among phenotype, functional activities, and morphology. Blood. 59:688-695.

4. Chan, W. C., S. Link, A. Mawle, I. Check, P. K. Brynes, and E. F. Winton. 1986. Heterogeneity of large granular lymphocyte proliferations. Delineation of two major subtypes. Blood. 68:1142-1153.

5. Semenzato, G., F. Pandolfi, T. Chisesi, D. E. Rossi, G. Pizzolo, R. Zambello, L. Trentin, C. Agostini, E. Dini, M. Vespignani, A. Cafaro, D. Pasgualetti, M. C. Giubellino, N. Migone, and R. Foa. 1987. The lymphoproliferative disease of granular lymphocytes. Cancer (Phila.). 60:2971-2978.

6. Epstein, M. A., and B. G. Achong. 1979. The Epstein-Barr virus. Springer-Verlag, Berlin. 1-22.

7. Rickinson, A. B. 1986. Chronic, symptomatic Epstein-Barr virus infection. Immunol. Today. 7:13-14.

8. Kawa-Ha, K., E. Franco, S. Doi, K. Yumura, S. Ishihara, A. Tawa, and H. Yabuuchi. 1987. Successful treatment of chronic active Epstein-Barr virus infection with recombinant interleukin-2. Lancet. i:154.

9. Aronson, F. R., R. A. Dempsey, M. Allegretta, J. AndreSchwartz, P. A. Poldre, C. D. Hillyer, D. R. Parkinson, R. A. Rudders, R. S. Schwartz, and J. W. Mier. 1987. Malignant granular lymphoproliferation after Epstein-Barr virus infection: partial immunologic reconstitution with interleukin-2. Am. J. Hematol. 25:427-439.

10. Oshimi, K. 1988. Granular lymphocytes proliferative disorders: report of 12 cases and review of the literature. Leukemia (Baltimore). 2:617-627.

11. Tagawa, S., Y. Tokumine, E. Ueda, K. Waki, Y. Kanayama, N. Taniguchi, T. Nakanishi, R. Inoue, and T. Kitani. 1986. Leu11+ Tr cell chronic lymphocytic leukemia with partially activated natural killer function and its further activation by recombinant IL2 in vitro. Blood. 68:846-852.

12. Tagawa, S., M. Hatakeyama, M. Shibano, T. Taniguchi, and T. Kitani. 1988. The expression of the p75 subunit of interleukin 2 receptor in Tac negative leukemic cells of two patients with large granular lymphocytic leukemia. Blood. 71:1161-1164.

13. Henle, W., G. Henle, and C. A. Horwitz. 1974. Epstein-Barr virus specific diagnostic tests in infectious mononucleosis. Hum. Pathol. 5:551-565.

14. Franco, E., K. Kawa-Ha, S. Doi, K. Yumura, M. Murata, S. Ishihara, A. Tawa, and H. Yabuuchi. 1987. Remarkable depression of $\mathrm{CD} 4+2 \mathrm{H} 4+\mathrm{T}$ cells in severe chronic active Epstein-Barr virus infection. Scand. J. Immunol. 26:769-773.

15. Ha-Kawa, K., K. Yumura, J. Hara, S. Ishihara, and H. Yabuuchi. 1987. Concomitant rearrangements of T-cell $\beta$ - and $\gamma$-chain genes in childhood T-lineage leukemia/lymphoma. Leuk. Res. 11:739-745.

16. Feinberg, A. P., and B. Vogelstein. 1983. A technique for radiolabeling DNA restriction endonuclease fragments to high specific activity. Anal. Biochem. 132:6-13.

17. Andiman, W., L. Gradoville, L. Heston, R. Neydorff, M. E. Savage, G. Kitchingman, D. Shedd, and G. Miller. 1983. Use of cloned probes to detect Epstein-Barr viral DNA in tissues of patients with neoplastic and lymphoproliferative diseases. J. Infect. Dis. 148:967977.

18. Shiraishi, Y., T. Taguchi, Y. Ohta, and K. Hirai. 1985. Chromosomal localization of the Epstein-Barr virus (EBV) genome in Bloom's syndrome B-lymphoblastoid cell lines transformed with EBV. Chromosoma (Berl.). 93:157-164.

19. Raab-Traub, N., and K. Flynn. 1986. The structure of the termini of the Epstein-Barr virus as a marker of clonal cellular proliferation. Cell. 47:883-889.

20. Brown, N. A., C. Liu, Y. Wang, and C. Garcia. 1988. B-cell lymphoproliferation and lymphomagenesis are associated with clonotypic intracellular terminal regions of the Epstein-Barr virus. J. Virol. 62:962-969.

21. Yanagi, Y., Y. Yoshikai, K. Leggett, S. P. Clark, I. Aleksander, and T. Mak. 1984. A human T cell-specific cDNA clone encodes a protein having extensive homology to immunoglobulin chains. Nature (Lond.). 308:145-149.

22. Lefranc, M. P., and J. H. Rabbitts. 1985 . Two tandemly organized human genes encoding the $T$-cell $\gamma$ constant-region sequences show multiple rearrangement in different T-cell types. Nature (Lond.). 316:464-466. 
23. Imamura, N., A. Kuramoto, K. Kawa-Ha, H. Fujii, and T. Takiguchi. 1988. Negative association between the human T-cell leukemia virus type 1 and large granular lymphocyte leukemia in Japan. Lancet. ii:962.

24. Nadler, L. M., P. Stashenko, R. Hardy, A. van Agthoven, C. Terhorst, and S. F. Schlossman. 1981. Characterization of a human B cell-specific antigen (B2) distinct from B1. J. Immunol. 126:19411947.

25. Weis, J. J., L. E. Toothaker, J. A. Smith, J. H. Weis, and D. T. Fearon. 1988. Structure of the human B lymphocyte receptor for C3d and the Epstein-Barr virus and relatedness to other members of the family of C3/C4 binding proteins. J. Exp. Med. 167:1047-1066.

26. Jones, J. F., S. Shurin, C. Abramowsky, R. R. Tubbs, C. G.
Sciotto, R. Wahl, J. Sands, D. Gottman, B. Z. Katz, and J. Sklar. 1988. T-cell lymphomas containing Epstein-Barr viral DNA in patients with chronic Epstein-Barr virus infections. N. Engl. J. Med. 318:733-741.

27. Kikuta, H., Y. Taguchi, K. Tomizawa, K. Kojima, N. Kawamura, A. Ishizaka, Y. Sakiyama, S. Matsumoto, S. Imai, T. Kinoshita, S. Koizumi, T. Osata, I. Kobayashi, I. Hamada, and K. Hirai. 1988. Epstein-Barr virus genome-positive $\mathrm{T}$ lymphocytes in a boy with chronic active EBV infection associated with kawasaki-like disease. Nature (Lond.). 333:455-457.

28. Ishihara, S., A. Tawa, K. Yumura-Yagi, M. Murata, J. Hara, H. Yabuuchi, K. Hirai, and K. Kawa-Ha. 1989. Clonal T-cell lymphoproliferation containing Epstein-Barr (EB) virus DNA in a patient with chronic active EB virus infection. Jpn. J. Cancer Res. (Gann). 80:99-101. 